\title{
Approximate Hamiltonian Symmetry Groups and Recursion Operators for Perturbed Evolution Equations
}

\author{
M. Nadjafikhah ${ }^{1}$ and A. Mokhtary ${ }^{2}$ \\ ${ }^{1}$ School of Mathematics, Iran University of Science and Technology, Narmak, Tehran 1684613114, Iran \\ ${ }^{2}$ Department of Complementary Education, Payame Noor University, Tehran 19395-3697, Iran \\ Correspondence should be addressed to A. Mokhtary; rdmokhtary@gmail.com
}

Received 11 October 2012; Revised 18 December 2012; Accepted 1 January 2013

Academic Editor: Rutwig Campoamor-Stursberg

Copyright (c) 2013 M. Nadjafikhah and A. Mokhtary. This is an open access article distributed under the Creative Commons Attribution License, which permits unrestricted use, distribution, and reproduction in any medium, provided the original work is properly cited.

\begin{abstract}
The method of approximate transformation groups, which was proposed by Baikov et al. (1988 and 1996), is extended on Hamiltonian and bi-Hamiltonian systems of evolution equations. Indeed, as a main consequence, this extended procedure is applied in order to compute the approximate conservation laws and approximate recursion operators corresponding to these types of equations. In particular, as an application, a comprehensive analysis of the problem of approximate conservation laws and approximate recursion operators associated to the Gardner equation with the small parameters is presented.
\end{abstract}

\section{Introduction}

The investigation of the exact solutions of nonlinear evolution equations has a fundamental role in the nonlinear physical phenomena. One of the significant and systematic methods for obtaining special solutions of systems of nonlinear differential equations is the classical symmetries method, also called Lie group analysis. This well-known approach originated at the end of nineteenth century from the pioneering work of Lie [1]. The fact that symmetry reductions for many PDEs cannot be determined, via the classical symmetry method, motivated the creation of several generalizations of the classical Lie group approach for symmetry reductions. Consequently, several alternative reduction methods have been proposed, going beyond Lie's classical procedure and providing further solutions. One of these techniques which is extremely applied particularly for nonlinear problems is perturbation analysis. It is worth mentioning that sometimes differential equations which appear in mathematical modelings are presented with terms involving a parameter called the perturbed term. Because of the instability of the Lie point symmetries with respect to perturbation of coefficients of differential equations, a new class of symmetries has been created for such equations, which are known as approximate (perturbed) symmetries. In the last century, in order to have the utmost result from the methods, combination of Lie symmetry method and perturbations are investigated and two different so-called approximate symmetry methods (ASMs) have been developed. The first method is due to Baikov et al. $[2,3]$. The second procedure was proposed by Fushchich and Shtelen [4] and later was followed by Euler et al. $[5,6]$. This method is generally based on the perturbation of dependent variables. In $[7,8]$, a comprehensive comparison of these two methods is presented.

As it is well known, Hamiltonian systems of differential equations are one of the famous and significant concepts in physics. These important systems appear in the various fields of physics such as motion of rigid bodies, celestial mechanics, quantization theory, fluid mechanics, plasma physics, and so forth. Due to the significance of Hamiltonian structures, in this paper, by applying the linear behavior of the Euler operator, characteristics, prolongation, and Fréchet derivative of vector fields, we have extended ASM on the Hamiltonian and bi-Hamiltonian systems of evolution equations, in order to investigate the interplay between approximate symmetry groups, approximate conservation laws, and approximate recursion operators. 
The structure of the present paper is as follows. In Section 2, some necessary preliminaries regarding to the Hamiltonian structures are presented. In Section 3, a comprehensive investigation of the approximate Hamiltonian symmetries and approximate conservation laws associated to the perturbed evolution equations is proposed. Also, as an application of this procedure, approximate Hamiltonian symmetry groups, approximate bi-Hamiltonian structures, and approximate conservation laws of the Gardner equation are computed. In Section 4, the approximate recursion operators are studied and the proposed technique is implemented for the Gardner equation as an application. Finally, some concluding remarks are mentioned at the end of the paper.

\section{Preliminaries}

In this section, we will mention some necessary preliminaries regarding Hamiltonian structures. In order to be familiar with the general concepts of the ASM, refer to [9]. It is also worth mentioning that most of this paper's definitions, theorems and techniques regarding Hamiltonian and biHamiltonian structures are inspired from [10].

Let $M \subset X \times U$ denote a fixed connected open subset of the space of independent and dependent variables $x=$ $\left(x^{1}, \ldots, x^{p}\right)$ and $u=\left(u^{1}, \ldots, u^{q}\right)$. The algebra of differential functions $P\left(x, u^{(n)}\right)=p[u]$ over $M$ is denoted by $\mathscr{A}$. We further define $\mathscr{A}^{l}$ to be the vector space of $l$-tuples of differential functions, $P[u]=\left(P_{1}[u], \ldots, P_{l}[u]\right)$, where each $P_{j} \in \mathscr{A}$.

A generalized vector field will be a (formal) expression of the form

$$
\mathbf{v}=\sum_{i=1}^{p} \xi^{i}[u] \frac{\partial}{\partial x^{i}}+\sum_{\alpha=1}^{q} \phi_{\alpha}[u] \frac{\partial}{\partial u^{\alpha}}
$$

in which $\xi^{i}$ and $\phi_{\alpha}$ are smooth differential functions. The Prolonged generalized vector field can be defined as follows:

$$
\mathbf{p r}^{(\mathbf{n})} \mathbf{v}=\mathbf{v}+\sum_{\alpha=1}^{q} \sum_{\sharp J \leq n} \phi_{\alpha}^{J}[u] \frac{\partial}{\partial u_{J}^{\alpha}},
$$

whose coefficients are determined by the formula

$$
\phi_{\alpha}^{J}=D_{J}\left(\phi_{\alpha}-\sum_{i=1}^{p} \xi^{i} u_{i}^{\alpha}\right)+\sum_{i=1}^{p} \xi^{i} u_{J, i}^{\alpha},
$$

with the same notation as before. Given a generalized vector field $\mathbf{v}$, its infinite prolongation (or briefly prolongation) is the formally infinite sum as follows:

$$
\boldsymbol{p r} \mathbf{v}=\sum_{i=1}^{p} \xi^{i} \frac{\partial}{\partial x^{i}}+\sum_{\alpha=1}^{q} \sum_{J} \phi_{\alpha}^{J} \frac{\partial}{\partial u_{J}^{\alpha}},
$$

where each $\phi_{\alpha}^{J}$ is given by (3), and the sum in (4) now extends over all multi-indices $J=\left(j_{1}, \ldots, j_{k}\right)$ for $k \geq 0,1 \leq j_{k} \leq p$.

A generalized vector field $\mathbf{v}$ is a generalized infinitesimal symmetry of a system of differential equations as follows:

$$
\Delta_{v}[u]=\Delta_{v}\left(x, u^{(n)}\right)=0, \quad v=1, \ldots, l,
$$

if and only if

$$
\operatorname{prv}\left[\Delta_{v}\right]=0, \quad v=1, \ldots, l,
$$

for every smooth solution $u=f(x)$.

Among all the generalized vector fields, those in which the coefficients $\xi^{i}[u]$ of the $\partial / \partial x^{i}$ are zero play a distinguished role. Let $Q[u]=\left(Q_{1}[u], \ldots, Q_{q}[u]\right) \in \mathscr{A}^{q}$ be a $q$-tuple of differential functions. The generalized vector field

$$
\mathbf{v}_{\mathrm{Q}}=\sum_{\alpha=1}^{q} Q_{\alpha}[u] \frac{\partial}{\partial u^{\alpha}}
$$

is called an evolutionary vector field, and $Q$ is called its characteristic.

A manifold $M$ with a Poisson bracket is called a Poisson manifold, the bracket defining a Poisson structure on $M$. Let $M$ be a Poisson manifold and $H: M \rightarrow \mathbb{R}$ be a smooth function. The Hamiltonian vector field associated with $H$ is the unique smooth vector field $\widehat{\mathbf{v}}_{\mathbf{H}}$ on $M$ satisfying the following identity:

$$
\widehat{\mathbf{v}}_{\mathbf{H}}=\{F, H\}=-\{H, F\}
$$

for every smooth function $F: M \rightarrow \mathbb{R}$. The equations governing the flow of $\widehat{\mathbf{v}}_{\mathbf{H}}$ are referred to as Hamilton's equations for the "Hamiltonian" function $H$.

Let $x=\left(x^{1}, \ldots, x^{m}\right)$ be local coordinates on $M$ and $H(x)$ be a real-valued function. The following basic formula can be obtained for the Poisson bracket:

$$
\{F, H\}=\sum_{i=1}^{m} \sum_{j=1}^{m}\left\{x^{i}, x^{j}\right\} \frac{\partial F}{\partial x^{i}} \frac{\partial H}{\partial x^{j}} .
$$

In other words, in order to compute the Poisson bracket of any pair of functions in some given set of local coordinates, it suffices to know the Poisson brackets between the coordinate functions themselves. These basic brackets,

$$
J^{i j}(x)=\left\{x^{i}, x^{j}\right\}, \quad i, j=1, \ldots, m,
$$

are called the structure functions of the Poisson manifold $M$ relative to the given local coordinates and serve to uniquely determine the Poisson structure itself. For convenience, we assemble the structure functions into a skew-symmetric $m \times$ $m$ matrix $J(x)$, called the structure matrix of $M$. Using $\nabla H$ to denote the (column) gradient vector for $H$, the local coordinate form (9) for the Poisson bracket can be written as

$$
\{F, H\}=\nabla F \cdot J \nabla H .
$$

Therefore, in the given coordinate chart, Hamilton's equations take the form of

$$
\frac{d x}{d t}=J(x) \nabla H(x) .
$$

Alternatively, using (9), we could write this in the "bracket form" as follows:

$$
\frac{d x}{d t}=\{x, H\}
$$


the $i$ th component of the right-hand side being $\left\{x^{i}, H\right\}$. A system of first-order ordinary differential equations is said to be a Hamiltonian system if there is a Hamiltonian function $H(x)$ and a matrix of functions $J(x)$ determining a Poisson bracket (11) whereby the system takes the form (12).

If

$$
\mathscr{D}=\sum_{J} P_{J}[u] D_{J}, \quad P_{J} \in \mathscr{A}
$$

is a differential operator, its (formal) adjoint is the differential operator $\mathscr{D}^{*}$ which satisfies

$$
\int_{\Omega} P \cdot \mathscr{D} Q d x=\int_{\Omega} Q \cdot \mathscr{D}^{*} P d x
$$

for every pair of differential functions $P, Q \in \mathscr{A}$ which vanish when $u \equiv 0$. Also, for every domain $\Omega \subset \mathbf{R}^{p}$ and every function $u=f(x)$ of compact support in $\Omega$. An operator $\mathscr{D}$ is self-adjoint if $\mathscr{D}^{*}=\mathscr{D}$; it is skew-adjoint if $\mathscr{D}^{*}=-\mathscr{D}$.

The principal innovations needed to convert a Hamiltonian system of ordinary differential equations (12) to a Hamiltonian system of evolution equations are as follows (refer to [10] for more details):

(i) replacing the Hamiltonian function $H(x)$ by a Hamiltonian functional $\mathscr{H}[u]$,

(ii) replacing the vector gradient operation $\nabla H$ by the variational derivative $\delta \mathscr{H}$ of the Hamiltonian functional, and

(iii) replacing the skew-symmetric matrix $J(x)$ by a skewadjoint differential operator $\mathscr{D}$ which may depend on $u$.

The resulting Hamiltonian system will take the form of

$$
\frac{\partial u}{\partial t}=\mathscr{D} \cdot \delta \mathscr{H}[u] .
$$

Clearly, for a candidate Hamiltonian operator $\mathscr{D}$ the correct expression for the corresponding Poison bracket has the form of

$$
\{\mathscr{P}, \mathscr{L}\}=\int \delta \mathscr{P} \cdot \mathscr{D} \delta \mathscr{L} d x,
$$

whenever $\mathscr{P}, \mathscr{L} \in \mathscr{F}$ are functionals. Off course, the Hamiltonian operator $\mathscr{D}$ must satisfy certain further restrictions in order that (17) be a true Poisson bracket. A linear operator $\mathscr{D}: \mathscr{A}^{q} \rightarrow \mathscr{A}^{q}$ is called Hamiltonian if its Poisson bracket (17) satisfies the conditions of skew-symmetry and the Jacobi identity.

Proposition 1. Let $\mathscr{D}$ be a Hamiltonian operator with Poisson bracket (17). To each functional $\mathscr{H}=\int H d x \in \mathscr{F}$, there is an evolutionary vector field pr $\widehat{\mathbf{v}}_{\mathscr{H}}$, called the Hamiltonian vector field associated with $\mathscr{H}$, which for all functionals $\mathscr{P} \in \mathscr{F}$ satisfies the following identity:

$$
\operatorname{pr} \widehat{\mathbf{v}}_{\mathscr{H}}(\mathscr{P})=\{\mathscr{P}, \mathscr{H}\} .
$$

Indeed, $\widehat{\mathbf{v}}_{\mathscr{H}}$ has characteristic $\mathscr{D} \delta \mathscr{H}=\mathscr{D E}(H)$, in which $\mathbf{E}$ is Euler operator (Proposition 7.2 of [10]).

\section{Approximate Hamiltonian Symmetries and Approximate Conservation Laws}

Consider a system of perturbed evolution equations:

$$
\frac{\partial u}{\partial t}=P[u, \varepsilon]
$$

in which $P[u, \varepsilon]=P\left(x, u^{(n)}, \varepsilon\right) \in \mathscr{A}^{q}, x \in \mathbb{R}^{p}, u \in \mathbb{R}^{q}$ and $\varepsilon$ is a parameter.

Substituting according to (19) and its derivatives, we see that any evolutionary symmetry must be equivalent to one whose characteristic $Q[u, \varepsilon]=Q\left(x, t, u^{(m)}, \varepsilon\right)$ depends only on $x, t, u, \varepsilon$ and the $x$-derivatives of $u$. On the other hand, (19) itself can be considered as the equations corresponding to the flow $\exp \left(t \mathbf{v}_{p}\right)$ of the evolutionary vector field with characteristic $P$. The symmetry criterion (6), which in this case is

$$
D_{t} Q_{v}=\operatorname{pr} \mathbf{v}_{Q}\left(P_{v}\right)+o\left(\varepsilon^{p}\right), \quad v=1, \ldots, q,
$$

can be readily seen to be equivalent to the following Lie bracket condition on the two approximate generalized vector fields. Indeed, this point generalizes the correspondence between symmetries of systems of first-order perturbed ordinary differential equations and the Lie bracket of the corresponding vector fields.

Considering the above assumptions, some useful relevant theorems and definitions could be rewritten as follows.

Proposition 2. An approximate evolutionary vector field $\mathbf{v}_{\mathrm{Q}}$ is a symmetry of the system of perturbed evolution equations $u_{t}=P[u, \varepsilon]$ if and only if

$$
\frac{\partial \mathbf{v}_{Q}}{\partial t}+\left[\mathbf{v}_{P}, \mathbf{v}_{Q}\right]=o\left(\varepsilon^{p}\right)
$$

holds identically in $\left(x, t, u^{(m)}, \varepsilon\right)$. (Here $\partial \mathbf{v}_{Q} / \partial t$ denotes the evolutionary vector field with characteristic $\partial Q / \partial t$.).

Any approximate conservation law of a system of perturbed evolution equations takes the form of

$$
D_{t} T+\operatorname{Div} X=o\left(\varepsilon^{p}\right),
$$

in which Div denotes spatial divergence. Without loss of generality, the conserved density $T\left(x, t, u^{(n)}, \varepsilon\right)$ can be assumed to depend only on $x$-derivatives of $u$. Equivalently, for $\Omega \subset X$, the functional

$$
\mathscr{T}[t ; u, \varepsilon]=\int_{\Omega} T\left(x, t, u^{(n)}, \varepsilon\right) d x
$$

is a constant, independent of $t$, for all solutions $u$ such that $T\left(x, t, u^{(n)}, \varepsilon\right) \rightarrow 0$ as $x \rightarrow \partial \Omega$. Note that if $T\left(x, t, u^{(n)}, \varepsilon\right)$ is any such differential function, and $u$ is a solution of the perturbed evolutionary system $u_{t}=P[u, \varepsilon]$, then

$$
D_{t} T \approx \partial_{t} T+\operatorname{pr} \mathbf{v}_{p}(T)
$$


where $\partial_{t}=\partial / \partial t$ denotes the partial $t$-derivative. Thus $T$ is the density for a conservation law of the system if and only if its associated functional $\mathscr{T}$ satisfies the following identity:

$$
\frac{\partial \mathscr{T}}{\partial t}+\operatorname{pr} \mathbf{v}_{p}(\mathscr{T})=o\left(\varepsilon^{p}\right) .
$$

In the case that our system is of Hamiltonian form, the bracket relation (18) immediately leads to the Noether relation between approximate Hamiltonian symmetries and approximate conservation laws.

Definition 3. Let $\mathscr{D}$ be a $q \times q$ approximate Hamiltonian differential operator. An approximate distinguished functional for $\mathscr{D}$ is a functional $\mathscr{G} \in \mathscr{F}$ satisfying $\mathscr{D} \delta \mathscr{G}=o\left(\varepsilon^{p}\right)$ for all $x, u$.

In other words, the Hamiltonian system corresponding to a distinguished functional is completely trivial: $u_{t}=0$.

Now, according to [10], the perturbed Hamiltonian version of Noether's theorem can be presented as follows.

Theorem 4. Let $u_{t}=\mathscr{D} \delta \mathscr{H}$ be a Hamiltonian system of perturbed evolution equations. An approximate Hamiltonian vector field $\widehat{\mathbf{v}}_{\mathscr{P}}$ with characteristic $\mathscr{D} \delta \mathscr{P}, \mathscr{P} \in \mathscr{F}$ determines an approximate generalized symmetry group of the system if and only if there is an equivalent functional $\widetilde{P} \approx \mathscr{P}-\mathscr{G}$ differing only from $\mathscr{P}$ by a time-dependent approximate distinguished functional $\mathscr{G}[t ; u, \varepsilon]$, such that $\widetilde{\mathscr{P}}$ determines an approximate conservation law.

Example 5. The Gardner equation

$$
u_{t}=6\left(u+\varepsilon u^{2}\right) u_{x}-u_{x x x}
$$

can in fact be written in Hamiltonian form in two distinct ways. Firstly, we see

$$
u_{t}=D_{x}\left(3 u^{2}+2 \varepsilon u^{3}-u_{x x}\right)=\mathscr{D} \delta \mathscr{H}_{1},
$$

where $\mathscr{D}=D_{x}$ and

$$
\mathscr{H}_{1}[u, \varepsilon]=\int\left(u^{3}+\frac{\varepsilon}{2} u^{4}+\frac{u_{x}^{2}}{2}\right) d x
$$

is an approximate conservation law. Note that $\mathscr{D}$ is certainly skew-adjoint and Hamiltonian. The Poisson bracket is

$$
\{\mathscr{P}, \mathscr{L}\}=\int \delta \mathscr{P} \cdot D_{x}(\delta \mathscr{L}) d x
$$

The second Hamiltonian form is

$$
u_{t}=\left(4 u D_{x}+2 u_{x}+3 \varepsilon\left(u u_{x}+u^{2} D_{x}\right)-D_{x}^{3}\right) u=\mathscr{E} \delta \mathscr{H}_{0},
$$

in which

$$
\mathscr{H}_{0}[u, \varepsilon]=\int \frac{1}{2} u^{2} d x
$$

$\mathscr{E}$ is skew-adjoint and satisfies the Jacobi identity. Therefore it is Hamiltonian.

In [11], we have comprehensively analyzed the problem of approximate symmetries for the Gardner equation. We have shown that the approximate symmetries of the Gardner equation are given by the following generators:

$$
\begin{gathered}
\mathbf{v}_{1}=\partial_{x}, \\
\mathbf{v}_{2}=\partial_{t}, \\
\mathbf{v}_{3}=6 t \partial_{x}+(2 \varepsilon u-1) \partial_{u}, \\
\mathbf{v}_{4}=\varepsilon \mathbf{v}_{1}, \\
\mathbf{v}_{5}=\varepsilon \mathbf{v}_{2}, \\
\mathbf{v}_{6}=\varepsilon\left(6 t \partial_{x}-\partial_{u}\right)=\varepsilon \mathbf{v}_{3}, \\
\mathbf{v}_{7}=\varepsilon\left(x \partial_{x}+3 t \partial_{t}-2 u \partial_{u}\right),
\end{gathered}
$$

with corresponding characteristics

$$
\begin{gathered}
Q_{1}=u_{x}, \\
Q_{2}=6\left(u+\varepsilon u^{2}\right) u_{x}-u_{x x x}, \\
Q_{3}=6 t u_{x}+1-2 \varepsilon u, \\
Q_{4}=\varepsilon Q_{1}=\varepsilon u_{x}, \\
Q_{5}=\varepsilon Q_{2}=\varepsilon\left(6 u u_{x}-u_{x x x}\right), \\
Q_{6}=\varepsilon Q_{3}=\varepsilon\left(6 t u_{x}+1\right), \\
Q_{7}=\varepsilon\left(2 u+x u_{x}+3 t\left(6 u u_{x}-u_{x x x}\right)\right),
\end{gathered}
$$

(up to sign).

For the first Hamiltonian operator $\mathscr{D}=D_{x}$, there is one independent nontrivial approximate distinguished functional, the mass $\mathscr{P}_{0}=\mathscr{M}=\int u d x$ which is consequently approximately conserved.

For the above seven characteristics, we have

$$
Q_{i} \approx D_{x} \delta \mathscr{P}_{i}, \quad i=1,2,4,5,6,
$$

with the following approximately conserved functionals:

$$
\begin{gathered}
\mathscr{P}_{1}=\mathscr{H}_{0}[u, \varepsilon]=\int \frac{1}{2} u^{2} d x, \\
\mathscr{P}_{2}=\mathscr{H}_{1}[u, \varepsilon]=\int\left(u^{3}+\frac{\varepsilon}{2} u^{4}+\frac{1}{2} u_{x}^{2}\right) d x, \\
\mathscr{P}_{4}=\varepsilon \mathscr{P}_{1}=\int \frac{\varepsilon}{2} u^{2} d x, \\
\mathscr{P}_{5}=\varepsilon \mathscr{P}_{2}=\varepsilon \int\left(u^{3}+\frac{1}{2} u_{x}^{2}\right) d x, \\
\mathscr{P}_{6}=\varepsilon \int\left(3 t u^{2}+x u\right) d x .
\end{gathered}
$$


For the second Hamiltonian operator $\mathscr{E}=4 u D_{x}+2 u_{x}+$ $3 \varepsilon\left(u u_{x}+u^{2} D_{x}\right)-D_{x}^{3}$,

$$
Q_{i} \approx \mathscr{E} \delta \widetilde{\mathscr{P}}_{i}, \quad i=2,4,5,7,
$$

the following approximately conserved functionals are the corresponding approximate conservation laws:

$$
\begin{gathered}
\widetilde{\mathscr{P}}_{2}=\mathscr{P}_{1}=\int \frac{1}{2} u^{2} d x, \\
\widetilde{\mathscr{P}}_{4}=\frac{\varepsilon}{2} \mathscr{P}_{0}=\frac{\varepsilon}{2} \int u d x, \\
\widetilde{\mathscr{P}}_{5}=\varepsilon \widetilde{\mathscr{P}}_{2}=\varepsilon \int \frac{1}{2} u^{2} d x, \\
\widetilde{\mathscr{P}}_{7}=\frac{1}{2} \mathscr{P}_{6}=\frac{\varepsilon}{2} \int\left(3 t u^{2}+x u\right) d x .
\end{gathered}
$$

In this case, nothing new is obtained. Note that the other approximate conservation law $\mathscr{P}_{5}$ did not arise from one of the geometrical symmetries. According to Theorem 4, however, there is an approximate Hamiltonian symmetry which gives rises to it, namely $\widehat{\mathbf{v}}_{\mathscr{P}_{5}}$. The characteristic of this approximate generalized symmetry is

$$
\begin{aligned}
\bar{Q}_{5} & \approx \mathscr{E} \delta \mathscr{P}_{5}=\mathscr{E} \varepsilon\left(3 u^{2}-u_{x x}\right) \\
& \approx \varepsilon\left(u_{x x x x x}-10 u u_{x x x}-20 u_{x} u_{x x}+30 u^{2} u_{x}\right) .
\end{aligned}
$$

Note that $\bar{Q}_{5}$ happens to satisfy the Hamiltonian condition (34) for $\mathscr{D}$ with the following functional:

$$
\overline{\mathscr{P}}_{5}=\frac{\varepsilon}{2} \int\left(u_{x x}^{2}-5 u^{2} u_{x x}+5 u^{4}\right) d x .
$$

Consequently, another approximate conservation law is provided for the Gardner equation.

Keeping on this procedure recursively, further approximate conservation laws could be generated. But, this procedure will be done in the next section by applying approximate recursion operators.

\section{Approximate Recursion Operators}

Definition 6. Let $\Delta$ be a system of perturbed differential equations. An approximate recursion operator for $\Delta$ is a linear operator $\mathscr{R}: \mathscr{A}^{q} \rightarrow \mathscr{A}^{q}$ in the space of $q$-tuples of differential functions with the property that whenever $\mathbf{v}_{Q}$ is an approximate evolutionary symmetry of $\Delta$, so $\mathbf{v}_{\widetilde{Q}}$ is with $\widetilde{Q} \approx \mathscr{R} Q$.

For nonlinear perturbed systems, there is an analogous criterion for a differential operator to be an approximate recursion operator, but to state it we need to introduce the notion of the (formal) Fréchet derivative of a differential function.

Definition 7. Let $P[u, \varepsilon]=P\left(x, u^{(n)}, \varepsilon\right) \in \mathscr{A}^{r}$ be an $r$-tuple of differential functions. The Fréchet derivative of $P$ is the perturbed differential operator $\mathbf{D}_{P}: \mathscr{A}^{q} \rightarrow \mathscr{A}^{r}$ defined so that

$$
\mathbf{D}_{P}(Q)=\left.\frac{d}{d \epsilon}\right|_{\epsilon=0} P[u+\epsilon Q[u, \varepsilon]]
$$

for any $Q \in \mathscr{A}^{q}$.

Proposition 8. If $P \in \mathscr{A}^{r}$ and $Q \in \mathscr{A}^{q}$ then

$$
\mathbf{D}_{P}(Q) \approx \operatorname{pr} \mathbf{v}_{Q}(P)
$$

Theorem 9. Suppose that $\Delta[u, \varepsilon]=0$ be a system of perturbed differential equations. If $\mathscr{R}: \mathscr{A}^{q} \rightarrow \mathscr{A}^{q}$ is a linear operator such that for all solutions $u$ of $\Delta$,

$$
\mathbf{D}_{\Delta} \cdot \mathscr{R} \approx \widetilde{\mathscr{R}} \cdot \mathbf{D}_{\Delta}
$$

where $\mathscr{R}: \mathscr{A}^{q} \rightarrow \mathscr{A}^{q}$ is a linear differential operator, then $\mathscr{R}$ is an approximate recursion operator for the system.

Suppose that $\Delta[u, \varepsilon]=u_{t}-K[u, \varepsilon]$ is a perturbed evolution equation. Then $\mathbf{D}_{\Delta}=D_{t}-\mathbf{D}_{K}$. If $\mathscr{R}$ is an approximate recursion operator, then it is not hard to observe that the operator $\widetilde{\mathscr{R}}$ in (42) must be the same as $\mathscr{R}$. Therefore, the condition (42) in this case reduces to the commutator condition

$$
\mathscr{R}_{t} \approx\left[\mathbf{D}_{K}, \mathscr{R}\right]
$$

for an approximate recursion operator of a perturbed evolution equation.

From (4), we can conclude that if $\mathscr{R}$ is an approximate recursion operator, then for all $l \geq 1$ in which $\varepsilon^{l} \mathscr{R} \neq 0, \varepsilon^{l} \mathscr{R}$ is an approximate recursion operator as follows:

$$
\left(\varepsilon^{l} \mathscr{R}\right)_{t}=\varepsilon^{l} \mathscr{R}_{t} \approx \varepsilon^{l}\left[\mathbf{D}_{K}, \mathscr{R}\right] \approx\left[\mathbf{D}_{K}, \varepsilon^{l} \mathscr{R}\right] .
$$

In order to illustrate the significance of the above theorem, we discuss a couple of examples, including the potential Burgers' equation and the Gardner equation. In the first example, we apply some technical methods, used in Examples 5.8 and 5.30 of $[10]$.

Example 10. Consider the potential Burgers' equation

$$
u_{t}=u_{x x}+\varepsilon u_{x}^{2}
$$


As mentioned in [7], approximate symmetries of the potential Burgers' equation are given by the following twelve vector fields

$$
\begin{gathered}
\mathbf{v}_{1}=\partial_{x}, \\
\mathbf{v}_{2}=\partial_{t}, \\
\mathbf{v}_{3}=x \partial_{x}+2 t \partial_{t}, \\
\mathbf{v}_{4}=2 t \partial_{x}-\left(x u-\varepsilon t \frac{u^{2}}{2}\right) \partial_{u}, \\
\mathbf{v}_{5}=\left(u-\varepsilon t \frac{u^{2}}{2}\right) \partial_{u}, \\
\mathbf{v}_{6}=4 x t \partial_{x}+4 t^{2} \partial_{t}-\left(x^{2}+2 t\right)\left(u-\varepsilon t \frac{u^{2}}{2}\right) \partial_{u}, \\
\mathbf{v}_{7}=\varepsilon \mathbf{v}_{1}, \\
\mathbf{v}_{8}=\varepsilon \mathbf{v}_{2}, \\
\mathbf{v}_{9}=\varepsilon\left(x \partial_{x}+2 t \partial_{t}\right)=\varepsilon \mathbf{v}_{3}, \\
\mathbf{v}_{10}=\varepsilon\left(2 t \partial_{x}-x u \partial_{u}\right)=\varepsilon \mathbf{v}_{4}, \\
\mathbf{v}_{11}=\varepsilon u \partial_{u}=\varepsilon \mathbf{v}_{5}, \\
\mathbf{v}_{12}=\varepsilon\left(4 x t \partial_{x}+4 t^{2} \partial_{t}-\left(x^{2}+2 t\right) u \partial_{u}\right)=\varepsilon \mathbf{v}_{6},
\end{gathered}
$$

plus the infinite family of vector fields

$$
\mathbf{v}_{f, g}=(f(x, t)(1-\varepsilon u)+\varepsilon g(x, t)) \partial_{u},
$$

where $f, g$ are arbitrary solutions of the heat equation $u_{t}=$ $u_{x x}$.

The corresponding characteristics of the first twelve approximate symmetries are

$$
\begin{aligned}
& Q_{1}=u_{x} \\
& Q_{2}=u_{x x}+\varepsilon u_{x}^{2} \\
& Q_{3}=x u_{x}+2 t\left(u_{x x}+\varepsilon u_{x}^{2}\right) \text {, } \\
& Q_{4}=x u+2 t u_{x}-\varepsilon t \frac{u^{2}}{2}, \\
& Q_{5}=u-\varepsilon t \frac{u^{2}}{2}, \\
& Q_{6}=\left(x^{2}+2 t\right)\left(u-\varepsilon t \frac{u^{2}}{2}\right)+4 x t u_{x}+4 t^{2}\left(u_{x x}+\varepsilon u_{x}^{2}\right) \text {, } \\
& Q_{7}=\varepsilon Q_{1}=\varepsilon u_{x}, \\
& Q_{8}=\varepsilon Q_{2}=\varepsilon u_{x x} \text {, } \\
& Q_{9}=\varepsilon Q_{3}=\varepsilon\left(x u_{x}+2 t u_{x x}\right) \text {, } \\
& Q_{10}=\varepsilon Q_{4}=\varepsilon\left(x u+2 t u_{x}\right) \text {, } \\
& Q_{11}=\varepsilon Q_{5}=\varepsilon u, \\
& Q_{12}=\varepsilon Q_{6}=\varepsilon\left(\left(x^{2}+2 t\right) u+4 x t u_{x}+4 t^{2} u_{x x}\right) \text {, }
\end{aligned}
$$

(up to sign).
Inspection of $Q_{1}, Q_{2}, Q_{7}, Q_{8}$ leads us to the conjecture that $\mathscr{R}_{1}=D_{x}+\varepsilon u_{x}$ is an approximate recursion operator, since $Q_{3}=\mathscr{R}_{1} Q_{1}, Q_{8}=\mathscr{R}_{1} Q_{7}$, and so forth. To prove this, we note that the Fréchet derivative for the right-hand side of potential Burgers' equation is

$$
D_{K}=D_{x}^{2}+2 \varepsilon u_{x} D_{x} .
$$

We must verify (4). The time derivative of the first approximate recursion operator $\mathscr{R}_{1}$ on the solutions of the potential Burgers' equation is the multiplication operator as follows:

$$
\left(\mathscr{R}_{1}\right)_{t}=\left(D_{x}+\varepsilon u_{x}\right)_{t}=\varepsilon u_{x t}=\varepsilon\left(u_{x x x}+2 \varepsilon u_{x} u_{x x}\right)=\varepsilon u_{x x x} .
$$

On the other hand, the commutator is computed using Leibniz' rule for differential operators:

$$
\left[D_{K}, \mathscr{R}_{1}\right]=\varepsilon u_{x x x} .
$$

Comparing these two verifies (4) and proves that $\mathscr{R}_{1}$ is an approximate recursion operator for the potential Burgers' equation.

There is thus an infinite hierarchy of approximate symmetries, with characteristics $\mathscr{R}_{1}^{k} Q_{1}, k=0,1,2, \ldots$ For example, the next characteristic after $Q_{12}$ in the sequence is

$$
\mathscr{R}_{1} Q_{12}=\varepsilon\left(\left(x^{2}+6 t\right) u_{x}+2 x\left(u+2 t u_{x x}\right)+4 t^{2} u_{x x x}\right) \text {. }
$$

To obtain the characteristics depending on $x$ and $t$, we require a second approximate recursion operator, which by inspection, we guess to be

$$
\mathscr{R}_{2}=t \mathscr{R}_{1}+\frac{x}{2}
$$

Using the fact that $\mathscr{R}_{1}$ satisfies (4), we readily find

$$
\left(\mathscr{R}_{2}\right)_{t}=t\left(\mathscr{R}_{1}\right)_{t}+\mathscr{R}_{1}=t\left[D_{K}, \mathscr{R}_{1}\right]+\mathscr{R}_{1},
$$

whereas

$$
\begin{aligned}
{\left[D_{K}, \mathscr{R}_{2}\right] } & =t\left[D_{K}, \mathscr{R}_{1}\right]+\left[D_{x}^{2}+2 \varepsilon u_{x} D_{x}, \frac{1}{2} x\right] \\
& =t\left[D_{K}, \mathscr{R}_{1}\right]+\left(D_{x}+\varepsilon u_{x}\right)=t\left[D_{K}, \mathscr{R}_{1}\right]+\mathscr{R}_{1},
\end{aligned}
$$

proving that $\mathscr{R}_{2}$ is also an approximate recursion operator. There is thus a doubly infinite hierarchy of approximate generalized symmetries of potential Burgers' equation, with characteristics $\mathscr{R}_{2}^{l} \mathscr{R}_{1}^{k} Q_{1}, k, l \geq 0$. For instance, $Q_{2}=\mathscr{R}_{1} Q_{1}$, $Q_{3}=2 \mathscr{R}_{2} \mathscr{R}_{1} Q_{1}$ and so on.

Example 11. Consider the Gardner equation, which was shown to have two Hamiltonian structures with

$$
\mathscr{D}=D_{x}, \quad \mathscr{E}=4 u D_{x}+2 u_{x}+3 \varepsilon\left(u u_{x}+u^{2} D_{x}\right)-D_{x}^{3} .
$$

Hence, the operator connecting our hierarchy of approximate Hamiltonian symmetries is

$$
\mathscr{R}=\mathscr{E} \cdot \mathscr{D}^{-1}=4 u+3 \varepsilon u^{2}+(2+3 \varepsilon u) u_{x} D_{x}^{-1}-D_{x}^{2} .
$$


Therefore, our results on approximate bi-Hamiltonian systems will provide ready-made proofs of the existence of infinitely many approximate conservation laws and approximate symmetries for the Gardner equation.

Note that the Fréchet derivative for the right-hand side of Gardner's equation is

$$
\begin{gathered}
\mathbf{D}_{K}=6(1+2 \varepsilon u) u_{x}+6\left(u+\varepsilon u^{2}\right) D_{x}-D_{x}^{3}, \\
\mathscr{R}_{t}=(4+6 \varepsilon u) u_{t}+\left(2 u_{x t}+3 \varepsilon u_{t} u_{x}+3 \varepsilon u u_{x t}\right) D_{x}^{-1} \\
=12 u u_{x}(2+5 \varepsilon u)-(4+6 \varepsilon u) u_{x x x} \\
+\left(6 u u_{x x}(2+5 \varepsilon u)+12 u_{x}^{2}(1+5 \varepsilon u)\right. \\
\left.-u_{x x x x}(2+3 \varepsilon u)-3 \varepsilon u_{x} u_{x x x}\right) D_{x}^{-1} .
\end{gathered}
$$

Theorem 12. Let $\bar{Q}_{0}=\varepsilon u_{x}$. For each $k \geq 0$, the differential polynomial $\bar{Q}_{k}=\mathscr{R}^{k} \bar{Q}_{0}$ is a total $x$-derivative, $\bar{Q}_{k}=D_{x} R_{k}$, and hence we can recursively define $\bar{Q}_{k+1}=\mathscr{R} \bar{Q}_{k}$. Each $\bar{Q}_{k}$ is the characteristic of an approximate symmetry of the Gardner equation.

Proof. To prove this theorem, we apply the similar method applied in Theorem 5.31 of [10].

We proceed by induction on $k$, so suppose that $\bar{Q}_{k}=$ $\mathscr{R}^{k} \bar{Q}_{0}$ for some $R_{k} \in \mathscr{A}$. From the form of the approximate recursion operator,

$$
\begin{aligned}
\bar{Q}_{k+1} & =\varepsilon\left(4 u \bar{Q}_{k}+2 u_{x} D_{x}^{-1} \bar{Q}_{k}-D_{x}^{2} \bar{Q}_{k}\right) \\
& =\varepsilon D_{x}\left(2 u D_{x}^{-1} \bar{Q}_{k}+2 D_{x}^{-1}\left(u \bar{Q}_{k}\right)-D_{x} \bar{Q}_{k}\right) .
\end{aligned}
$$

If we can prove that for some differential polynomial $S_{k} \in \mathscr{A}$, $u \bar{Q}_{k}=D_{x} S_{k}$, we will indeed have proved that $\bar{Q}_{k+1}=D_{x} R_{k+1}$, where $R_{k+1}$ is the above expression in brackets. Consequently, the induction step will be completed.

To prove this fact, note that the formal adjoint of the approximate recursion operator $\varepsilon \mathscr{R}$ is

$$
\varepsilon \mathscr{R}^{*}=\varepsilon\left(4 u-2 D_{x}^{-1} \cdot u_{x}-D_{x}^{2}\right)=D_{x}^{-1} \varepsilon \mathscr{R} D_{x} .
$$

We apply this in order to integrate the expression $u \bar{Q}_{k}$, by parts, so

$$
\bar{Q}_{k}=u \mathscr{R}^{k}\left[\varepsilon u_{x}\right]=u_{x} \cdot\left(\varepsilon \mathscr{R}^{*}\right)^{k}[u]+D_{x} A_{k}
$$

for some differential function $A_{k} \in \mathscr{A}$. On the other hand, using a further integration by parts, for some $B_{k} \in \mathscr{A}$ the following identity holds:

$$
\begin{aligned}
u_{x} \cdot\left(\varepsilon \mathscr{R}^{*}\right)^{k}[u] & =u_{x} \cdot D_{x}^{-1} \varepsilon \mathscr{R}\left[u_{x}\right] \\
& =u_{x} \cdot D_{x}^{-1} \bar{Q}_{k} \\
& =-u \bar{Q}_{k}+D_{x} B_{k} .
\end{aligned}
$$

Substituting into the previous identity, we conclude

$$
u \bar{Q}_{k}=D_{x} S_{k}, \quad \text { where } S_{k}=\frac{1}{2}\left(A_{k}+B_{k}\right),
$$

which proves our claim.
Definition 13. A pair of skew-adjoint $q \times q$ matrix of differential operators $\mathscr{D}$ and $\mathscr{E}$ is said to form an approximately Hamiltonian pair if every linear combination $a \mathscr{D}+$ $b \mathscr{E}, a, b \in \mathbb{R}$, is an approximate Hamiltonian operator. A system of perturbed evolution equations is an approximate biHamiltonian system if it can be written in the form of

$$
\frac{\partial u}{\partial t}=K_{1}[u, \varepsilon] \approx \mathscr{D} \delta \mathscr{H}_{1} \approx \mathscr{E} \delta \mathscr{H}_{0}
$$

where $\mathscr{D}, \mathscr{E}$ form an approximately Hamiltonian pair, and $\mathscr{H}_{0}$ and $\mathscr{H}_{1}$ are appropriate Hamiltonian functionals.

Lemma 14. If $\mathscr{D}, \mathscr{E}$ are skew-adjoint operators, then they form an approximately Hamiltonian pair if and only if $\mathscr{D}, \mathscr{E}$ and $\mathscr{D}+\mathscr{E}$ are all approximate Hamiltonian operators.

Corollary 15. Let $\mathscr{D}$ and $\mathscr{E}$ be Hamiltonian differential operators. Then $\mathscr{D}, \mathscr{E}$ form an approximately Hamiltonian pair if and only if

$$
\operatorname{pr} \mathbf{v}_{\mathscr{D} \theta}\left(\Theta_{\mathscr{C}}\right)+\operatorname{pr} \mathbf{v}_{\mathscr{C} \theta}\left(\Theta_{\mathscr{D}}\right)=o\left(\varepsilon^{p}\right),
$$

where

$$
\Theta_{\mathscr{D}}=\frac{1}{2} \int\{\theta \wedge \mathscr{D} \theta\} d x, \quad \Theta_{\mathscr{E}}=\frac{1}{2} \int\{\theta \wedge \mathscr{E} \theta\} d x
$$

are the functional bi-vectors representing the respective Poisson brackets.

Example 16. Consider the approximate Hamiltonian operators $\mathscr{D}, \mathscr{E}$ associated with the Gardner equation. We have

$$
\begin{aligned}
\operatorname{pr} \mathbf{v}_{\mathscr{D} \theta} & =\sum_{\alpha, J} D_{J}(\mathscr{D} \theta)_{\alpha} \frac{\partial}{\partial u_{J}^{\alpha}} \\
& =\sum_{\alpha, J} D_{J}\left(\sum_{\beta=1}^{q} \mathscr{D}_{\alpha \beta} \theta^{\beta}\right) \frac{\partial}{\partial u_{J}^{\alpha}}
\end{aligned}
$$

in the case of the second approximate Hamiltonian operator for the Gardner equation, we have

$$
\begin{gathered}
\operatorname{pr} \mathbf{v}_{\mathscr{E} \theta}(u)=\mathscr{E} \theta, \quad \operatorname{pr} \mathbf{v}_{\mathscr{E} \theta}\left(u^{2}\right)=2 u \mathscr{E} \theta, \\
\operatorname{pr} \mathbf{v}_{\mathscr{E} \theta}\left(\Theta_{\mathscr{D}}\right)=\operatorname{pr} \mathbf{v}_{\mathscr{E} \theta} \int \frac{1}{2}\left\{\theta \wedge \theta_{x}\right\} d x=o\left(\varepsilon^{p}\right)
\end{gathered}
$$

trivially, by the properties of the wedge product, it is deduced that

$$
\begin{aligned}
\operatorname{pr} & \mathbf{v}_{\mathscr{D} \theta}\left(\Theta_{\mathscr{C}}\right) \\
& =\operatorname{pr} \mathbf{v}_{\mathscr{D} \theta} \int\left\{\left(2 u+\frac{3 \varepsilon}{2} u^{2}\right) \theta \wedge \theta_{x}+\frac{1}{2} \theta_{x} \wedge \theta\right\} d x \\
& \approx \int\left\{(2+3 \varepsilon u) \theta_{x} \wedge \theta \wedge \theta_{x}\right\}=o\left(\varepsilon^{p}\right) .
\end{aligned}
$$

Thus $\mathscr{D}$ and $\mathscr{E}$ form an approximately Hamiltonian pair.

Definition 17. A differential operator $\mathscr{D}: \mathscr{A}^{r} \rightarrow \mathscr{A}^{s}$ is approximately degenerate if there is a nonzero differential operator $\widetilde{\mathscr{D}}: \mathscr{A}^{\mathcal{S}} \rightarrow \mathscr{A}$ such that $\widetilde{\mathscr{D}} \cdot \mathscr{D} \equiv o\left(\varepsilon^{p}\right)$. 
Now, according to [10], we are in a situation to state the main theorem on approximate bi-Hamiltonian systems.

Theorem 18. Let

$$
u_{t}=K_{1}[u, \varepsilon] \approx \mathscr{D} \delta \mathscr{H}_{1} \approx \mathscr{E} \delta \mathscr{H}_{0}
$$

be an approximate bi-Hamiltonian system of perturbed evolution equations. Assume that the operator $\mathscr{D}$ of the approximately Hamiltonian pair is approximate nondegenerate. Let $\mathscr{R}=\mathscr{E} \cdot \mathscr{D}^{-1}$ be the corresponding approximate recursion operator, and let $K_{0} \approx \mathscr{D} \delta \mathscr{H}_{0}$. Assume that for each $n=$ $1,2, \ldots$ one can recursively define

$$
K_{n} \approx \mathscr{R} K_{n-1}, \quad n \geq 1,
$$

meaning that for each $n, K_{n-1}$ lies in the image of $\mathscr{D}$. Then there exists a sequence of functionals $\mathscr{H}_{0}, \mathscr{H}_{1}, \mathscr{H}_{2}, \ldots$ such that

(i) for each $n \geq 1$ the perturbed evolution equation

$$
u_{t}=K_{n}[u, \varepsilon] \approx \mathscr{D} \delta \mathscr{H}_{n} \approx \mathscr{E} \delta \mathscr{H}_{n-1}
$$

is an approximate bi-Hamiltonian system;

(ii) the corresponding approximate evolutionary vector fields $\mathbf{v}_{n}=\mathbf{v}_{K_{n}}$ all mutually commute

$$
\left[\mathbf{v}_{n}, \mathbf{v}_{m}\right]=o\left(\varepsilon^{p}\right), \quad n, m \geq 0
$$

(iii) the approximate Hamiltonian functionals $\mathscr{H}_{n}$ are all in involution with respect to either Poisson bracket:

$$
\left\{\mathscr{H}_{n}, \mathscr{H}_{n}\right\}_{\mathscr{D}}=o\left(\varepsilon^{p}\right)=\left\{\mathscr{H}_{n}, \mathscr{H}_{n}\right\}_{\mathscr{E}}, \quad n, m \geq 0,
$$

and hence provide an infinite collection of approximate conservation laws for each of the approximate biHamiltonian systems (63).

We have seen that given an approximate bi-Hamiltonian system, the operator $\mathscr{R}=\mathscr{E} \cdot \mathscr{D}^{-1}$, when applied successively to the initial equation $K_{0}=\mathscr{D} \delta \mathscr{H}_{0}$, produces an infinite sequence of approximate generalized symmetries of the original system (subject to the technical assumptions contained in Theorem 18). It is still not clear that $\mathscr{R}$ is a true approximate recursion operator for the system, in the sense that whenever $\mathbf{v}_{\mathrm{Q}}$ is an approximate generalized symmetry, so is $\mathbf{v}_{\mathscr{R Q}}$. So far, we only know it for approximate symmetries with $Q=K_{n}$ for some $n$. In order to establish this more general result, we need a formula for the infinitesimal change of the approximate Hamiltonian operator itself under a Hamiltonian flow.

Lemma 19. Let $u_{t}=K \approx \mathscr{D} \delta \mathscr{H}$ be an approximate Hamiltonian system of perturbed evolution equations with corresponding vector field $\mathbf{v}_{K}=\widehat{\mathbf{v}}_{\mathscr{H}}$. Then

$$
\operatorname{pr} \widehat{\mathbf{v}}_{\mathscr{H}}(\mathscr{D}) \approx \mathbf{D}_{K} \cdot \mathscr{D}+\mathscr{D} \cdot \mathbf{D}_{K}^{*}
$$

Theorem 20. Let $u_{t}=K \approx \mathscr{D} \delta \mathscr{H}_{1} \approx \mathscr{E} \delta \mathscr{H}_{0}$ be an approximate bi-Hamiltonian system of perturbed evolution equations. Then the operators $\mathscr{R}_{l}=\varepsilon^{l} \mathscr{E} \cdot \mathscr{D}^{-1}, 0 \leq l \leq p$, are approximate recursion operators for the system.
Judging from $\mathscr{R}_{l}^{p}=o\left(\varepsilon^{p}\right)$, when $l \neq 0$, this type of approximate recursion operators have less significance than $\mathscr{R}_{0}$.

Example 21. The approximate recursion operators of the Gardner equation are

$$
\begin{gathered}
\mathscr{R}_{0}=\mathscr{E} \cdot \mathscr{D}^{-1}=4 u+2 u_{x} D_{x}^{-1}+3 \varepsilon\left(u u_{x} D_{x}^{-1}+u^{2}\right)-D_{x}^{2}, \\
\mathscr{R}_{1}=\varepsilon\left(4 u+2 u_{x} D_{x}^{-1}-D_{x}^{2}\right)
\end{gathered}
$$

and we can apply $\mathscr{R}_{0}$ to the right-hand side of the Gardner equation to obtain the approximate symmetries. The first step in this recursion is the flow

$$
\begin{aligned}
u_{t} \approx & \mathscr{E} \delta \mathscr{H}_{1} \approx \mathscr{D} \delta \mathscr{H}_{2} \\
\approx & u_{x x x x x}-10 u u_{x x x}-20 u_{x} u_{x x}+30 u^{2} u_{x} \\
& +\varepsilon\left(55 u^{3} u_{x}-39 u u_{x} u_{x x}-9 u^{2} u_{x x x}-12 u_{x}^{3}\right),
\end{aligned}
$$

which is not approximately total derivative, so we cannot reapply the approximate recursion operator to get a meaningful approximate generalized symmetry.

But if we set

$$
\begin{aligned}
& \bar{K}_{1}[u, \varepsilon]=Q_{5}=\varepsilon K_{1}[u, \varepsilon]=\varepsilon\left(6 u u_{x}-u_{x x x}\right), \\
& \overline{\mathscr{H}}_{0}=\widetilde{\mathscr{P}}_{5}=\varepsilon \mathscr{H}_{0}, \quad \overline{\mathscr{H}}_{1}=\mathscr{P}_{5}=\varepsilon \mathscr{H}_{1},
\end{aligned}
$$

then we can apply $\mathscr{R}_{0}$ successively to $\bar{K}_{1}$ in order to obtain the approximate symmetries. The first phase become

$$
\begin{aligned}
u_{t} & \approx \mathscr{E} \delta \overline{\mathscr{H}}_{1} \approx \mathscr{D} \delta \overline{\mathscr{H}}_{2} \approx \mathscr{R}_{0} \bar{K}_{1} \\
& \approx \varepsilon\left(u_{x x x x x}-10 u u_{x x x}-20 u_{x} u_{x x}+30 u^{2} u_{x}\right)
\end{aligned}
$$

in which

$$
\overline{\mathscr{H}}_{2}=\overline{\mathscr{P}}_{5}=\frac{\varepsilon}{2} \int\left(u_{x x}^{2}-5 u^{2} u_{x x}+5 u^{4}\right) d x
$$

is another approximate conservation law.

Now, for $\bar{K}_{2}=\mathscr{R}_{0} \bar{K}_{1}$ we have

$$
\begin{aligned}
u_{t} \approx & \mathscr{E} \delta \overline{\mathscr{H}}_{2} \approx \mathscr{D} \delta \overline{\mathscr{H}}_{3} \approx \mathscr{R}_{0} \bar{K}_{2} \\
\approx & \varepsilon\left(-u_{x x x x x x x}+14 u u_{x x x x x}+42 u_{x} u_{x x x x}\right) \\
& +70 \varepsilon\left(u_{x x} u_{x x x}-u^{2} u_{x x x}+2 u^{3} u_{x}-4 u u_{x} u_{x x}-u_{x}^{3}\right)
\end{aligned}
$$

where

$$
\overline{\mathscr{H}}_{3}=7 \varepsilon \int\left(\frac{u_{x x x}^{2}}{14}+u u_{x x}^{2}+5 u^{2} u_{x}^{2}+u^{5}\right) d x
$$

is a further approximate conservation law. 


\section{Concluding Remarks}

Sometimes, differential equations appearing in mathematical modelings are written with terms involving a small parameter which is known as the perturbed term. Taking into account the instability of the Lie point symmetries with respect to perturbation of coefficients of differential equations, the approximate (perturbed) symmetries for such equations are obtained. Different methods for computing the approximate symmetries of a system of differential equations are available in the literature [2-4].

The approximate symmetry method proposed by Fushchich and Shtelen [4] is based on a perturbation of dependent variables. This method has so many advantages such as producing more approximate group-invariant solutions, consistence with the perturbation theory, solving singular perturbation problems $[7,8]$, and close relationship with approximate homotopy symmetry method [12]. But despite the above-mentioned benefits, this procedure converts a perturbed evolution equation to an equivalent perturbed evolutionary system. In his case, obtaining the corresponding Hamiltonian formulation will be hard. Due to the increase of the dimensions of Hamiltonian operators $\mathscr{D}, \mathscr{E}$, computation of the approximate recursion operator $\mathscr{R}=\mathscr{E} \cdot \mathscr{D}^{-1}$ is difficult.

Since prolongation and Fréchet derivative of vector fields are linear, both of the approximate symmetry methods can be extended on the Hamiltonian structures. But due to the significance of vector fields in Hamiltonian and biHamiltonian systems, the approximate symmetry method proposed by Baikov et al. [2,3] seems to be more consistent.

\section{Acknowledgments}

It is a pleasure to thank the anonymous referees for their constructive suggestions and helpful comments which have improved the presentation of the paper. The authors wish to express their sincere gratitude to Fatemeh Ahangari for her useful advice and suggestions.

\section{References}

[1] S. Lie, "On integration of a class of linear partial differential equations by means of definite integrals," Archiv der Mathematik, vol. 6, pp. 328-368, 1881.

[2] V. A. Baikov, R. K. Gazizov, and N. Kh. Ibragimov, "Approximate symmetries of equations with a small parameter," Matematicheskil Sbornik, vol. 136, no. 4, pp. 435-450, 1988.

[3] V. A. Baikov, R. K. Gazizov, and N. H. Ibragimov, "Approximate transformation groups and deformations of symmetry Lie algebras," in CRC Handbook of Lie Group Analysis of Differential Equations, N. H. Ibragimov, Ed., vol. 3, chapter 2, CRC Press, Boca Raton, Fla, USA, 1996.

[4] W. I. Fushchich and W. M. Shtelen, "On approximate symmetry and approximate solutions of the nonlinear wave equation with a small parameter," Journal of Physics A, vol. 22, no. 18, pp. L887L890, 1989.
[5] N. Euler, M. W. Shul'ga, and W.-H. Steeb, "Approximate symmetries and approximate solutions for a multidimensional LandauGinzburg equation," Journal of Physics A, vol. 25, no. 18, pp. L1095-L1103, 1992.

[6] M. Euler, N. Euler, and A. Köhler, "On the construction of approximate solutions for a multidimensional nonlinear heat equation," Journal of Physics A, vol. 27, no. 6, pp. 2083-2092, 1994.

[7] M. Pakdemirli, M. Yürüsoy, and İ. T. Dolapçi, "Comparison of approximate symmetry methods for differential equations," Acta Applicandae Mathematicae, vol. 80, no. 3, pp. 243-271, 2004.

[8] R. Wiltshire, "Two approaches to the calculation of approximate symmetry exemplified using a system of advection-diffusion equations," Journal of Computational and Applied Mathematics, vol. 197, no. 2, pp. 287-301, 2006.

[9] N. H. Ibragimov and V. F. Kovalev, Approximate and Renormgroup Symmetries, Nonlinear Physical Science, Springer, Berlin, Germany, 2009.

[10] P. J. Olver, Applications of Lie Groups to Differential Equations, vol. 107 of Graduate Texts in Mathematics, Springer, New York, NY, USA, 2nd edition, 1993.

[11] M. Nadjafikhah and A. Mokhtary, "Approximate symmetry analysis of Gardner equation," http://arxiv.org/abs/1212.3604 .

[12] Z. Zhang, "Approximate homotopy series solutions of perturbed PDEs via approximate symmetrymethod," http://128.84. $158.119 / \mathrm{abs} / 1112.4225 \mathrm{v} 2$ 


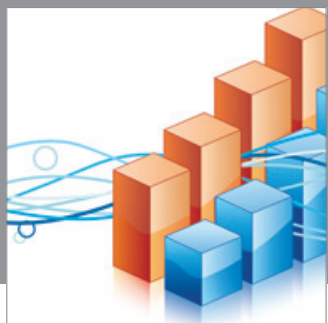

Advances in

Operations Research

mansans

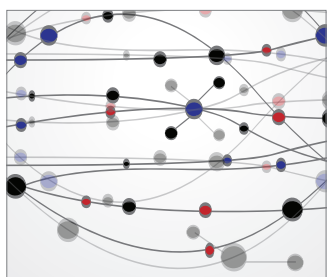

The Scientific World Journal
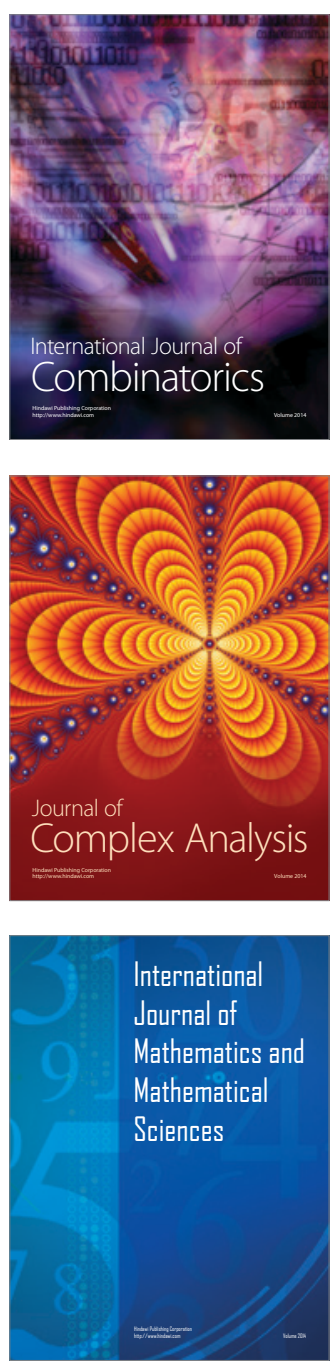
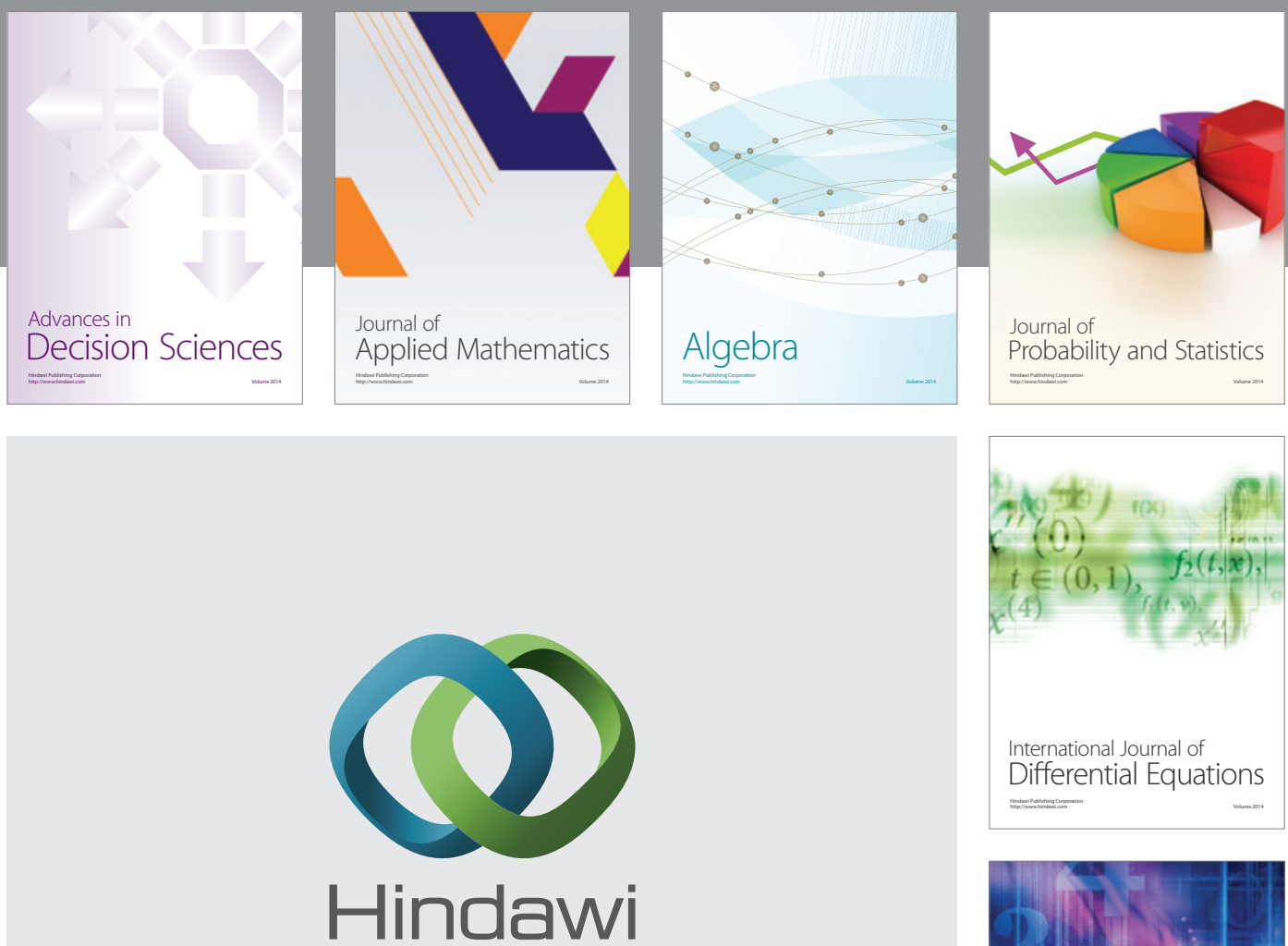

Submit your manuscripts at http://www.hindawi.com
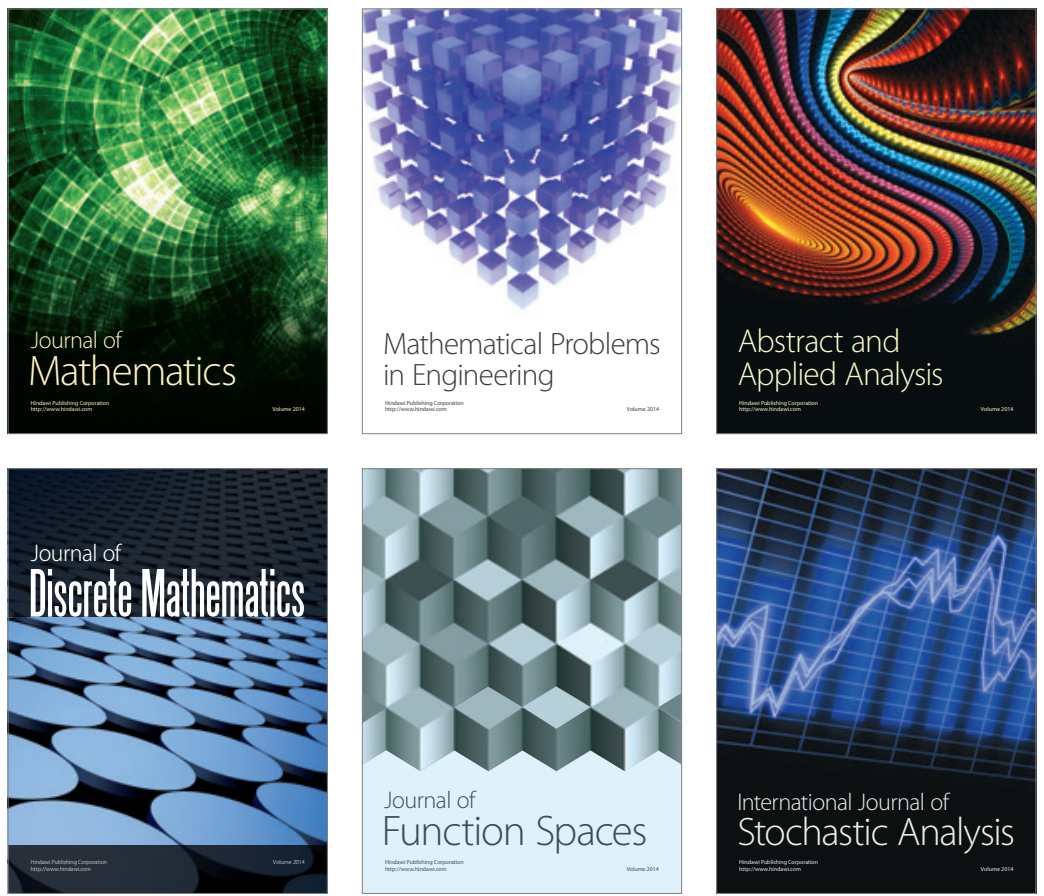

Journal of

Function Spaces

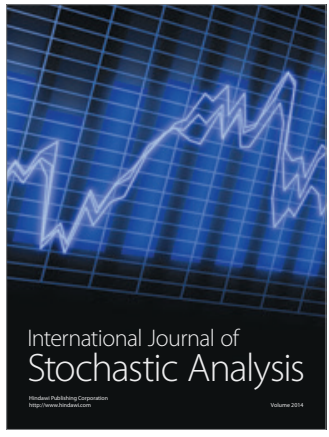

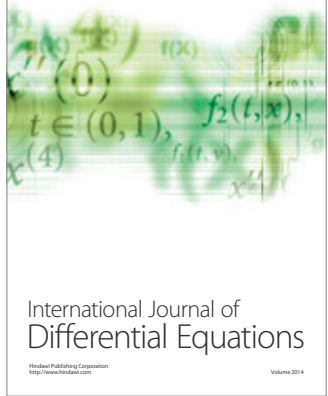
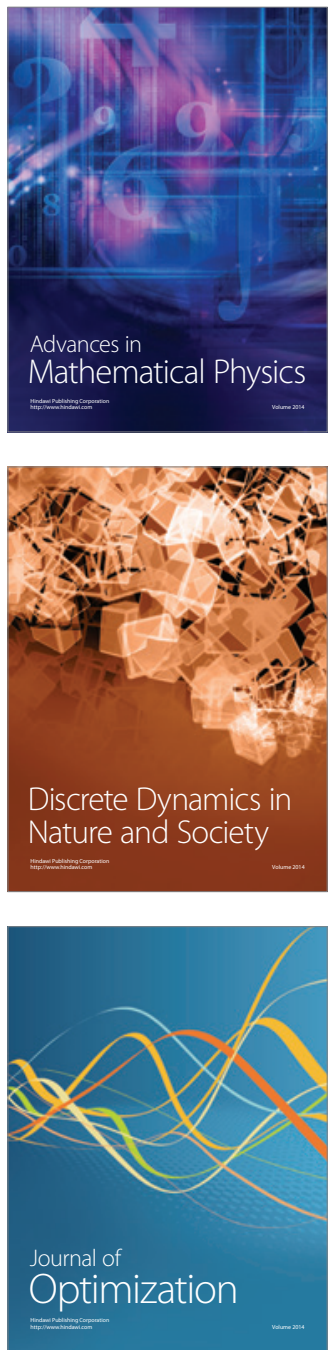